\title{
INCIDÊNCIA DE Cercosporidium personatum EM AMENDOIM SUBMETIDO A IRRIGAÇÃO PLENA E DEFICITÁRIA
}

Pablo Nascimento de Oliveira França ${ }^{1}$; Willians César Carrega ${ }^{2}$; Rogério Teixeira de Faria ${ }^{3}$; Anderson Prates Coelho ${ }^{4}$

${ }^{1}$ Mestrando em Agronomia (Ciência do Solo), FCAV/UNESP, Jaboticabal, SP, pnof@hotmail.com; ²Doutor em Agronomia (Produção Vegetal), FCAV/UNESP, Jaboticabal, SP; ${ }^{3}$ Docente da FCAV/UNESP, Jaboticabal, SP; ${ }^{4}$ Mestrando em Agronomia (Produção Vegetal), FCAV/UNESP, Jaboticabal, SP.

RESUMO: O déficit hídrico associado a presença de patógenos é um fator limitante no rendimento do cultivo de amendoim. Visando maior estabilidade da produtividade dessa cultura, estudos para determinação da quantidade ideal de água e avaliações sobre os impactos de doenças, como a manchapreta, é de fundamental importância. Diante disso, o objetivo desse trabalho foi avaliar os efeitos de cinco lâminas de irrigação e a incidência de mancha-preta no cultivo de amendoim. O experimento foi conduzido em delineamento em blocos casualizados, com tratamentos de 5 lâminas de irrigação $(\mathrm{L} 1=24 \% ; \mathrm{L} 2=47 \% ; \mathrm{L} 3=75 \% ; \mathrm{L} 4=100 \%$ e $\mathrm{L} 5=114 \%)$, calculadas em função de frações da evapotranspiração da cultura (ETc). No estágio R6, realizaram-se avaliações de danos da manchapreta, levando-se em conta a evolução da doença até o final do ciclo do cultivo. Utilizou-se a escala de notas variando de 1 a 9 , em que 1, representou a ausência de danos e 9 danos de $81 \%$ a $100 \%$. Verificou-se aumento linear na incidência da doença, sendo os danos visuais mais intensos para as menores lâminas de irrigação aplicadas (24\%, 47\% e 75\%). Concluiu-se que o déficit hídrico promove maior severidade de danos da mancha-preta, provavelmente devido à maior sensibilidade das plantas sob estresse hídrico.

Palavras-chave: Arachis hypogaea L., mancha-preta, déficit hídrico, produtividade.

\section{INTRODUÇÃO}

O déficit hídrico é um fator limitante no rendimento da cultura do amendoim (THANGTHONG et al., 2017). Kambiranda et al. (2011) citam uma estimativa anual que mostra que as perdas na produtividade de amendoim causada pela seca equivalem a US\$520 milhões. Associado ao déficit hídrico, a ocorrência de patógenos, pode comprometer ainda mais a produtividade do amendoim. Entre os patógenos, a mancha-preta causada por Cercosporidium personatum (Berk. \& Curt.) Deighton é considerada uma das principais doenças fúngicas que causam perdas na produção mundial de amendoim (CLEVENGER et al., 2018). Estima-se que em áreas de produção de amendoim sem pulverizações, as perdas no rendimento variam de 30\% a 70\% (SINGH et al., 2011).

Atualmente, poucos trabalhos foram realizados para avaliar o déficit hídrico em amendoim no Estado de São Paulo, principal produtor nacional. Visando maior sustentabilidade, estudos para 
determinação da quantidade ideal de água e avaliações sobre os impactos de doenças, como a manchapreta em diferentes lâminas de irrigação é de fundamental importância. Assim, objetivo desse trabalho foi avaliar os efeitos de lâminas de irrigação e a incidência de $C$. personatum em amendoim semeado na época de safrinha, de fevereiro a junho.

\section{MATERIAL E MÉTODOS}

O experimento foi conduzido na fazenda de ensino, pesquisa e extensão (FEPE), pertencente a Universidade Estadual Paulista "Júlio de Mesquita Filho" (UNESP), Câmpus de Jaboticabal, SP. A localização geográfica da área está definida pelas coordenadas $21^{\circ} 15^{\prime} 22^{\prime \prime}$ Latitude Sul e $48^{\circ} 18^{\prime} 58^{\prime \prime}$ Longitude Oeste, sendo a altitude média $570 \mathrm{~m}$ e declividade média do solo igual a 7\%, apresentando clima tipo Cwa (subtropical) de acordo com a classificação de Köeppen. O solo da área experimental é classificado como Latossolo Vermelho Eutroférrico típico, com textura muito argilosa, A moderado, caulinítico, relevo suave ondulado e ondulado (EMBRAPA 2013).

Semeou-se a cultivar IAC 505 que apresenta ciclo variando entre 130 e 140 dias (no estado de São Paulo), moderada resistência a doenças foliares e apresenta relativa tolerância à seca. A semeadura foi realizada no espaçamento de $0,9 \mathrm{~m}$ entre linhas e densidade média 15 plantas por $\mathrm{m}^{2}$. As sementes foram previamente tratadas com o fungicida thiram (Vitavax ${ }^{\circledR}$-Thiram $200 \mathrm{SC}, 250 \mathrm{~mL}$ do p.c. $100 \mathrm{~kg}^{-1}$ ) para evitar a incidência de patógenos. A semeadura ocorreu no dia 10 de fevereiro de 2018, ou seja, mais tardiamente, visando avaliar a produção de sementes fora da época das águas. No momento da semeadura, realizou-se adubação com a formula 0-20-20 (N, P, K) na quantidade de 300 $\mathrm{kg} \mathrm{ha}^{-1}$.

O delineamento experimental foi em blocos casualizados, tendo como tratamentos, 5 lâminas de irrigação, calculadas em função da reposição de frações da evapotranspiração de referência (ETc), correspondentes a $\mathrm{L} 1=24 \%$; L2 $=47 \%$; L3 $=75 \%$; L4 $=100 \%$ e $\mathrm{L} 5=114 \%$, obtidas semanalmente segundo a necessidade da cultura considerando o estádio fenológico e seu Kc. O experimento foi instalado em uma área $288 \mathrm{~m}^{2}$, incluindo 60 parcelas com quatro linhas/parcela de $6,24 \mathrm{~m}^{2}(2,4 \mathrm{~m} \mathrm{x}$ 2,6 m), sendo considerada como útil as duas linhas centrais das parcelas, medindo 2,24 $\mathrm{m}^{2}(1,4 \mathrm{~m} \mathrm{x}$ $1,6 \mathrm{~m})$.

Para determinação da ETo, utilizou-se a equação de Penman-Monteith (ALLEN et al., 1998), com base nos dados meteorológicos diários coletados na Estação Agroclimatológica da FCAVUNESP. Para irrigação nas parcelas utilizou-se um sistema de aspersão em linha, com aspersores Senninger (Modelo 3023-2 com duplo bocal de 8 x $5 \mathrm{~mm}$ ), operados com pressão de $300 \mathrm{kPa}$, no espaçamento $6 \mathrm{~m}$, produzindo um raio de alcance de $12 \mathrm{~m}$ e intensidade de aplicação de $13 \mathrm{~mm} \mathrm{~h}^{-1}$. Conforme a disposição das parcelas experimentais e o arranjo dos aspersores previamente descrito, e com base em testes realizados com coletores de precipitação, determinou-se o fator de distribuição do 
precipitado para obtenção da lâmina de irrigação aplicado em cada tratamento, seguindo metodologia proposta por DANTAS, 2015.

$\mathrm{Na}$ área experimental, realizou-se aplicações semanais rotacionando fungicidas com os seguintes ingredientes ativos: Pyraclostrobin + Epoxiconazole (Ópera $\left.{ }^{\circledR}\right)$, Bentazona (Basagran ${ }^{\circledR} 600$ ) e Ciproconazol (ALTO ${ }^{\circledR}$ 100), nas doses de 109,8 g i.a.ha ${ }^{-1} ; 720$ g i.a.ha ${ }^{-1}$ e 25 g i.a.ha ${ }^{-1}$, respectivamente. Mesmo com as aplicações semanais, houve alta incidência de mancha-preta. Por isso, realizou-se, a cada 10 dias, a partir do estádio reprodutivo R6, avaliações dos danos de C. personatum, atribuindo-se notas de sintomas visuais da doença por meio de uma escala variando de 1 a 9 , em que 1 , representa a ausência de danos e 9 danos de $81 \%$ a $100 \%$. Esta escala levou em consideração a quantidade de manchas/folha, o número de folhas com danos e a quantidade de desfolha ao longo do ramo. A nota foi aplicada em um dos ramos primários da planta, tomado aleatoriamente (SUBRAHMANYAM et al., 1982).

Os resultados obtidos foram submetidos a análise de variância pelo teste $\mathrm{F}$ e as médias comparadas pelo teste de Tukey a 5\% de probabilidade, utilizando o software Agrostat (BARBOSA \& MALDONADO JUNIOR, 2015).

\section{RESULTADOS E DISCUSSÃO}

Com base no teste de irrigação realizados com os aspersores, verificou-se que a distribuição gradual das lâminas se ajustou a curva polinomial, indicando que as parcelas estavam com as porcentagens d'água adequadas para realização do experimento, com $\mathrm{R}^{2}$ de 0,994 , como pode ser observado na Figura 1.

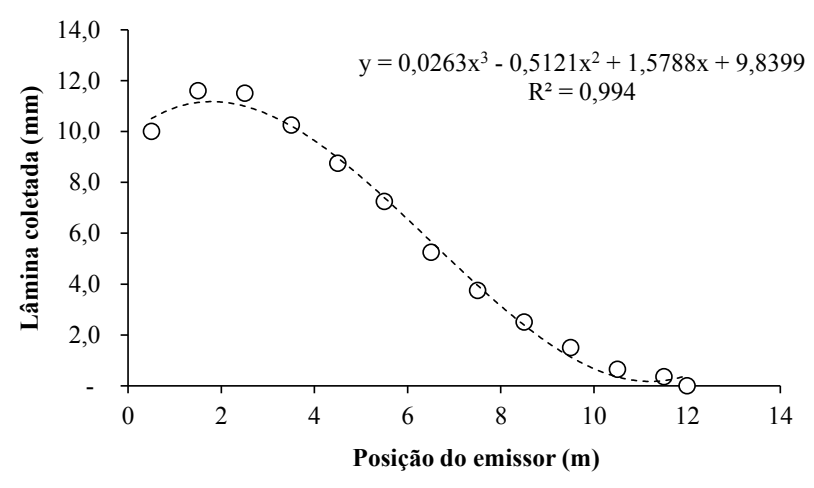

Figura 1. Distribuição gradual da lâmina de irrigação.

Para a mancha-preta, a partir do estádio reprodutivo R6, houve um aumento linear da incidência da doença, sendo os danos visuais mais intensos para as lâminas de irrigação que apresentavam os menores índices pluviométricos em todas as épocas de avaliação (Figura 2). 


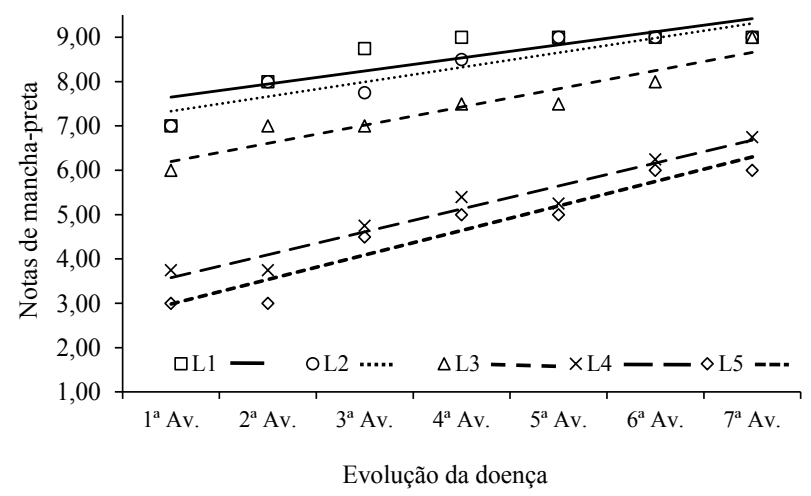

Figura 2. Evolução das notas de doenças causados pelo $C$. personatum entre as parcelas sob lâminas de irrigação. Equações: $\mathrm{L} 1, \mathrm{y}=0,295 \mathrm{x}+7,357, \mathrm{R}^{2}=0,70 ; \mathrm{L} 2, \mathrm{y}=0,330 \mathrm{x}+7,000, \mathrm{R}^{2}=0,85 ; \mathrm{L} 3, \mathrm{y}=$ $0,411 x+5,786 R^{2}=0,91 ; L 4, y=0,518 x+3,057, R^{2}=0,95 ; L 5, y=0,554 x+2,429, R^{2}=0,92$.

Comparando-se o efeito das lâminas de irrigação na incidência de C. personatum em cada época avaliada, verificou-se que houve diferença significativa entre os tratamentos hídricos (Tabela 1). Houve diferenças significativas entre os tratamentos L1 e L2 com os demais tratamentos. Verificou-se que logo após o início do estágio R6, houve aumento da severidade das doenças para os tratamentos com menores lâminas, apresentando notas visuais superiores a 7 (41\% a 60\% afetada). A partir dos 119 DAS, as plantas de amendoim apresentaram sintomas visuais da mancha-preta superiores a $90 \%$. Efeito semelhante foi constatado para o tratamento L3, contudo, verificou-se danos superiores a $90 \%$ apenas aos 147 DAS, 28 dias de diferença em relação ao tratamento L1 (Tabela 1).

Tabela 1. Comparação de notas de danos visuais de C. personatum para avaliações (Av.) realizadas a partir do estádio reprodutivo R6, em função de tratamentos de lâminas de irrigação aplicadas.

\begin{tabular}{|c|c|c|c|c|c|c|c|}
\hline Lâminas de irrigação & $1^{\mathrm{a}} \mathrm{Av}$. & $2^{\mathrm{a} A v .}$ & $3^{\mathrm{a} A v}$ & $4^{\mathrm{a}} \mathrm{Av}$. & $5^{\mathrm{a}} \mathrm{Av}$. & $6^{\mathrm{a}} \mathrm{Av}$. & $7^{\mathrm{a}} \mathrm{Av}$. \\
\hline L1 $-24 \%$ & $7,00 \mathrm{~A}$ & $8,00 \mathrm{~A}$ & $8,75 \mathrm{~A}$ & $9,00 \mathrm{~A}$ & $9,00 \mathrm{~A}$ & $9,00 \mathrm{~A}$ & $9,00 \mathrm{~A}$ \\
\hline L2 $-47 \%$ & $7,00 \mathrm{~A}$ & $8,00 \mathrm{~A}$ & $7,75 \mathrm{~A}$ & $8,50 \mathrm{~A}$ & $8,50 \mathrm{AB}$ & $9,00 \mathrm{~A}$ & $9,00 \mathrm{~A}$ \\
\hline $\mathrm{L} 3-75 \%$ & $6,00 \mathrm{~B}$ & $6,25 \mathrm{~A}$ & $7,00 \mathrm{~A}$ & $7,50 \mathrm{~B}$ & $6,75 \mathrm{BC}$ & $8,00 \mathrm{~A}$ & $9,00 \mathrm{~A}$ \\
\hline $\mathrm{L} 4-100 \%$ & $3,75 \mathrm{C}$ & $3,75 \mathrm{~B}$ & $5,00 \mathrm{~B}$ & $5,25 \mathrm{C}$ & $5,00 \mathrm{C}$ & $6,25 \mathrm{~B}$ & $6,75 \mathrm{~B}$ \\
\hline L5 - $114 \%$ & $3,00 \mathrm{D}$ & $3,00 \mathrm{~B}$ & $4,50 \mathrm{~B}$ & $5,00 \mathrm{C}$ & $5,00 \mathrm{C}$ & $5,25 \mathrm{~B}$ & $6,00 \mathrm{C}$ \\
\hline $\mathrm{F}$ & $279,00 * *$ & $20,72 * *$ & $21,49 * *$ & $116,14^{* *}$ & $20,29 * *$ & $23,95 * *$ & $171,00 * *$ \\
\hline C.V. $(\%)$ & 4,18 & 17,74 & 11,82 & 4,85 & 12,21 & 9,19 & 2,81 \\
\hline
\end{tabular}

Médias seguidas de mesma letra na coluna não diferem entre si ao nível de 5\% de probabilidade pelo Teste de Tukey. Pelo teste $\mathrm{F},{ }^{* *}$ significativo a $1 \%$ de probabilidade.

A severidade da doença foi expressiva, prejudicando drasticamente a planta de amendoim de forma mais acentuada nas menores lâminas aplicadas (tratamentos L1, L2 e L3). O déficit hídrico causado por esses tratamentos promoveu maior sensibilidade da planta de amendoim à doença. Plantas sob deficiência hídrica apresentam alterações nos processos morfológicos e fisiológicos, como o fechamento estomático, que reduz a transpiração, influenciando negativamente na taxa de absorção de $\mathrm{CO}_{2}$ pelas folhas para realização da fotossíntese (PEREIRA et al., 2016), reduzindo a área foliar (HARO et al., 2008) e, deixando as plantas mais suscetíveis a incidência de patógenos. Para as lâminas 
que receberam reposição de $100 \%$ e 114\% da necessidade da cultura (L4 e L5), a gravidade dos danos causados pela doença foi menor, não impedindo que atingissem o ciclo aos 147 DAS sem serem afetadas drasticamente pelo avanço da doença, completando normalmente o ciclo da cultura.

\section{CONCLUSÃO}

A partir do estágio reprodutivo R6, ocorre aumento linear na incidência de C. personatum para todas as lâminas de irrigação aplicadas na cultura do amendoim semeado tardiamente;

$\mathrm{O}$ déficit hídrico causado pelas lâminas de irrigação inferiores às necessidades hídricas da cultura (24\%, 47\% e 75\% da ETc) causa maior severidade de danos da mancha-preta, provavelmente devido à maior sensibilidade das plantas sob estresse hídrico.

\section{REFERÊNCIAS BIBLIOGRÁFICAS}

ALLEN, R. G.; PEREIRA, L. S.; RAES, D.; SMITH, M. Crop evapotranspiration - Guidelines for computing crop water requirements. Rome: FAO. 1998. 300 p.

BARBOSA, J. C.; MALDONADO JUNIOR, W. 2015. AgroEstat. Sistema para análises Estatísticas de ensaios agronômicos. Jaboticabal: FCAV/UNESP. 396p.

Clevenger, J.; CHU, Y.; ChAVARro, C.; BOtTON, S.; CUlBreAth, A.; ISLEIB, T. G.; HOLBROOK, C. C.; OZIAS-AKINS, P. Mapping Late Leaf Spot Resistance in Peanut (Arachis hypogaea) Using QTL-seq Reveals Markers for Marker-Assisted Selection. Frontiers in plant science, v. 9, p. 83, 2018.

DANTAS, G. F. Produtividade e qualidade da Brachiaria irrigada no outono/inverno. Dissertação (Mestrado em Agronomia-Ciência do Solo) - Universidade Estadual Paulista "Júlio de Mesquita Filho", Câmpus de Jaboticabal, 29 p., 2015.

EMPRESA BRASIILEIRA DE PESQUISA AGROPECUÁRIA. Sistema brasileiro de classificação de solos. 3. ed. Brasília, DF: Embrapa, 2013. 353 p.

HARO, R. J.; DARDANELLI, J. L.; OTEGUI, M. E.; COLLINO, D. J. Seed yield determination of peanut crops under water deficit: soil strength effects on pod set, the source-sink ratio and radiation use efficiency. Field Crops Research, v. 109, n. 1, p. 24-33, 2008.

KAMBIRANDA, D. M.; VASANTHAIAH, H. K.; KATAM, R.; ANANGA, A.; BASHA, S. M.; NAIK, K. Impact of drought stress on peanut (Arachis hypogaea L.) productivity and food safety. Plants and Environment. In Tech Publisher, p. 249-272, 2011.

PEREIRA, J. W. L.; ALBUQUeRQUe, M. B.; Melo FILHO, P. A.; NOGUEIRA, R. J. M. C.; LIMA, L. M.; SANTOS, R. C. Assessment of drought tolerance of peanut cultivars based on physiological and yield traits in a semiarid environment. Agricultural Water Management, v. 166, n. 1, p. 70-76, 2016. 
16 e 17 de agosto de 2018, centro de convenções da FCAV/UNESP - Câmpus de Jaboticabal, SP

SUBRAHMANYAM, P.; McDONALD, D.; GIBBONS. R.W.; NIGAM, S.N.; NEVILL, D.J. Resistance to rust and late leaf spot diseases in some genotypes of Arachis hypogaea. Peanut Science, v. 9, n. 1, p. 6-10, 1982.

SINGH, M. P.; ERICKSON, J. E.; BOOTE, K. J.; TILLMAN, B. J.; JONES, J. W.; VAN BRUGGEN, A. H. C. Late leaf spot e ects on growth, photosynthesis, and yield in peanut cultivars of di ering resistance. Agronomy journal, v. 103, n. 1, p. 85-91, 2011.

THANGTHONG, N.; JOGLOY, S.; JONGRUNGKLANG, N.; KVIEN, C. K.; PENSUK, V.; KESMALA, T.; VORASOOT, N. Root distribution patterns of peanut genotypes with different drought resistance levels under early season drought stress. Journal of Agronomy and Crop Science, v. 204, n. 2, p. 111-122, 2018. 